and $19 \%$ of women who did and did not receive the Pelvicol ${ }^{\circledR}$ implant, respectively $(P=0.019)$. Two women in each group experienced stage III anterior vaginal prolapse. Additionally, 5\% of women had posterior recurrence and 3\% had unsatisfactory results in the upper vaginal tract. The Pelvicol ${ }^{\circledR}$ implant was removed from one patient 1 month after surgery because it had extruded.

The authors conclude that the Pelvicol ${ }^{\circledR}$ implant reduces the incidence of recurrent anterior vaginal wall prolapse after colporrhaphy, and is well tolerated. They suggest extended follow-up studies of patients who receive this implant.

Original article Meschia M et al. (2007) Porcine skin collagen implants to prevent anterior vaginal wall prolapse recurrence: a multicenter, randomized study. J Urol 177: 192-195

\section{Surgery after multiple chemotherapy regimens benefits men with testicular cancer}

Although $20-30 \%$ of men with testicular cancer experience tumor progression or recurrence after first-line chemotherapy, 35-70\% of these patients will respond to salvage chemotherapy. Postchemotherapy resection of residual disease following multiple chemotherapy regimens remains, however, an important component of treatment for men with advanced testicular cancer.

Eggener and colleagues evaluated 71 men with testicular cancer who underwent two or more chemotherapy regimens followed by retroperitoneal lymph-node dissection (RPLND) to resect all known residual masses. These patients were followed for a median of 30 months (52 months for survivors), and the 5-year and 10-year disease-specific survival rates were $74 \%$ and $70 \%$, respectively. Almost half of the patients had either viable germ-cell tumor or teratoma at RPLND, but those who received taxane-based salvage chemotherapy regimens had a decreased rate of viable germcell tumor (14\% versus $42 \%$ ). Retroperitoneal and extraperitoneal histology were discordant in $25 \%$ of cases, which emphasized the need for resection of all residual masses.

Eggener and colleagues recommend postchemotherapy surgery for selected patients with advanced germ-cell tumors: RPLND for those who respond to salvage chemotherapy and lack radiologically visible residual disease; RPLND with extraperitoneal resection for patients who respond to chemotherapy and have resectable residual masses. Some patients with residual masses and elevated serum tumor markers might also benefit from postchemotherapy surgery.

Original article Eggener SE et al. (2007) Pathologic findings and clinical outcome of patients undergoing retroperitoneal lymph node dissection after multiple chemotherapy regimens for metastatic testicular germ cell tumors. Cancer 109: 528-535

\section{Y-chromosome loci could be involved in prostate-cancer risk}

Studies have suggested that genes on the $Y$ chromosome could have a role in increased susceptibility to prostate cancer. Men with mutations in such genes might have altered probabilities of having daughters or sons; Harlap and colleagues, therefore, investigated whether a man's risk of developing prostate cancer is related to the sex of his offspring.

The authors studied the relative risk (RR) of developing prostate cancer in a single population of 38,934 Israeli men who were followed up from the birth of their offspring (between 1964 and 1976) until 2005. In total, 712 men were diagnosed as having invasive prostate cancer. Men who only had daughters had a $40 \%$ increased risk of prostate cancer (adjusted RR 1.40, $P<0.001)$ compared with men who had at least one son. In men with one, two, three or more offspring, the RRs of developing prostate cancer associated with lack of sons were 1.25, 1.41 and 1.60, respectively. As the number of sons increased, the RR of prostate cancer decreased $(P<0.0001)$, but the number of daughters had no effect.

The hypothesis that $\mathrm{Y}$-chromosome loci are involved in prostate cancer in at least some men, seems to be borne out by these findings, although no information was available about offspring of these fathers born before or after the 13-year study period.

Further studies of male reproductive outcomes would be needed to provide additional information on the genes involved in prostate cancer.

Original article Harlap S et al. (2007) Prostate cancer in fathers with fewer male offspring: the Jerusalem perinatal study cohort. J Natl Cancer Inst 99: 77-81 